\title{
Erratum to: Melts of garnet lherzolite: experiments, models and comparison to melts of pyroxenite and carbonated lherzolite
}

\author{
Timothy L. Grove $\cdot$ Eva S. Holbig $\cdot$ Jay A. Barr • \\ Christy B. Till $\cdot$ Michael J. Krawczynski
}

Published online: 23 November 2014

(C) Springer-Verlag Berlin Heidelberg 2014

\section{Erratum to: Contrib Mineral Petrol (2013) 166:887-910 DOI 10.1007/s00410-013-0899-9}

An unfortunate coding error led to erroneous predicted abundances of $\mathrm{Na}_{2} \mathrm{O}$ in the melting models presented in Table 5 of Grove et al. (2013). The source of this error has been discovered and corrected; the cause of the discrepancy was an error in assignment of the partition coefficient expressions used for orthopyroxene and clinopyroxene. The values presented in a corrected Table 5 are the ones obtained using the appropriate partition coefficients for $\mathrm{Na}_{2} \mathrm{O}$. Our models of garnet lherzolite melts now have $\mathrm{Na}_{2} \mathrm{O}$ abundances that are similar to the melts predicted by Longhi (2002) and the experimentally produced melt of Walter (1998).
The online version of the original article can be found under doi:10.1007/s00410-013-0899-9.

T. L. Grove $(\bowtie) \cdot$ E. S. Holbig · J. A. Barr

Department of Earth, Atmospheric and Planetary Sciences,

Massachusetts Institute of Technology, Cambridge, MA 02139,

USA

e-mail: tlgrove@mit.edu

C. B. Till

US Geological Survey, Menlo Park, CA 94025, USA

\section{J. Krawczynski}

Department of Earth, Environmental and Planetary Sciences, Case Western Reserve University, Cleveland, OH 44106, USA
Table 5 Comparison of garnet lherzolite melting model of Longhi and test of model

\begin{tabular}{|c|c|c|c|c|c|c|c|c|}
\hline & \multicolumn{4}{|c|}{$\begin{array}{l}\text { Primitive H\&Z } 10 \text { and } 1 \% \\
\text { melts }\end{array}$} & \multicolumn{4}{|c|}{$\begin{array}{l}\text { Depleted H\&Z } 10 \text { and } 1 \% \\
\text { melts }\end{array}$} \\
\hline & $10 \%^{\mathrm{a}}$ & L’02 & $1 \%$ & L'02 & $10 \%$ & L’02 & $1 \%$ & L'02 \\
\hline $\mathrm{SiO}_{2}$ & 45.7 & 46.2 & 46.0 & 45.6 & 45.8 & 46.0 & 45.4 & 44.9 \\
\hline $\mathrm{TiO}_{2}$ & 1.14 & 0.98 & 2.09 & 1.43 & 1.04 & 0.97 & 2.09 & 1.61 \\
\hline $\mathrm{Al}_{2} \mathrm{O}_{3}$ & 13.0 & 13.0 & 14.9 & 15.1 & 12.8 & 12.8 & 13.1 & 14.3 \\
\hline $\mathrm{Cr}_{2} \mathrm{O}_{3}$ & 0.18 & 0.32 & 0.13 & 0.22 & 0.18 & 0.34 & 0.15 & 0.29 \\
\hline $\mathrm{FeO}$ & 10.8 & 9.60 & 9.66 & 9.60 & 10.6 & 9.70 & 10.6 & 10.1 \\
\hline $\mathrm{MgO}$ & 17.4 & 18.1 & 14.3 & 15.3 & 18.0 & 18.5 & 16.6 & 17.2 \\
\hline $\mathrm{CaO}$ & 9.58 & 9.76 & 7.85 & 6.91 & 9.86 & 10.1 & 8.48 & 8.34 \\
\hline $\mathrm{Na}_{2} \mathrm{O}$ & 1.96 & 1.57 & 2.82 & 2.68 & 1.80 & 1.32 & 2.93 & 1.96 \\
\hline \multirow[t]{2}{*}{$\mathrm{K}_{2} \mathrm{O}$} & 0.29 & 0.28 & 2.27 & 2.13 & 0.07 & 0.07 & 0.59 & 0.54 \\
\hline & \multicolumn{6}{|c|}{$40.07^{b}$} & \multicolumn{2}{|r|}{ Model $^{\mathrm{c}}$} \\
\hline $\mathrm{SiO}_{2}$ & \multicolumn{6}{|c|}{45.5} & \multicolumn{2}{|r|}{45.6} \\
\hline $\mathrm{TiO}_{2}$ & \multicolumn{6}{|c|}{1.27} & \multicolumn{2}{|c|}{0.89} \\
\hline $\mathrm{Al}_{2} \mathrm{O}_{3}$ & \multicolumn{6}{|c|}{10.3} & \multicolumn{2}{|r|}{11.1} \\
\hline $\mathrm{Cr}_{2} \mathrm{O}_{3}$ & \multicolumn{6}{|c|}{0.25} & \multicolumn{2}{|c|}{0.16} \\
\hline $\mathrm{FeO}$ & \multicolumn{6}{|c|}{10.7} & \multicolumn{2}{|c|}{10.9} \\
\hline $\mathrm{MgO}$ & \multicolumn{6}{|c|}{19.9} & \multicolumn{2}{|r|}{20.0} \\
\hline $\mathrm{CaO}$ & \multicolumn{6}{|c|}{9.31} & \multicolumn{2}{|r|}{9.70} \\
\hline $\mathrm{Na}_{2} \mathrm{O}$ & \multicolumn{6}{|c|}{1.08} & \multicolumn{2}{|r|}{0.94} \\
\hline $\mathrm{K}_{2} \mathrm{O}$ & \multicolumn{6}{|c|}{0.7} & \multicolumn{2}{|c|}{0.65} \\
\hline$T\left({ }^{\circ} \mathrm{C}\right)$ & \multicolumn{6}{|c|}{1,610} & \multicolumn{2}{|r|}{1,624} \\
\hline
\end{tabular}

Model calculation at $3 \mathrm{GPa}$ and 10 and $1 \%$ melting from this study and Longhi (2002)

a Melts of Hart and Zindler primitive (Hart and Zindler 1986) and depleted (Kinzler and Grove 1992) mantle compositions

b 40.07 shows the composition and temperature of a Lherzolite melt from Walter (1998) at $4 \mathrm{GPa}$ and $13 \mathrm{wt} \%$ melting

c Forward model of melting using the Walter (1998) lherzolite composition and $13 \%$ melting at $4 \mathrm{GPa}$. Temperature is that predicted by the model 


\section{References}

Grove TL, Holbig ES, Barr JA, Till CB, Krawczynski MJ (2013) Mantle melting in the garnet stability field: experiments and predictive models. Contrib Mineral Petrol 166:887-910. doi:10.1007/s00410-013-0899-9

Hart SR, Zindler A (1986) In search of a bulk-Earth composition. Chem Geol 57(3-4):247-267
Kinzler RJ, Grove TL (1992) Primary magmas of midocean ridge basalts 2. Applications. J Geophys Res 97(B5):6907-6926

Longhi J (2002) Some phase equilibrium systematics of lherzolite melting: I. Geochem Geophys Geosyst 3:art. no.-1020

Walter MJ (1998) Melting of garnet peridotite and the origin of komatiite and depleted lithosphere. J Petrol 39(1):29-60 